\title{
Thermal Modeling of Carbon-Epoxy Laminates in Fire Environments
}

\author{
MATTHEW T. McGURN ${ }^{1}$, PAUL E. DESJARDIN ${ }^{1}$, and AMANDA B. DODD ${ }^{2}$ \\ ${ }^{1}$ Department of Mechanical and Aerospace Engineering \\ University at Buffalo, the State University of New York (SUNY Buffalo) \\ Buffalo, NY 14260-4400 \\ ${ }^{2}$ Sandia National Laboratories \\ Albuquerque, NM 87185
}

\begin{abstract}
A thermal model is developed for the response of carbon-epoxy composite laminates in fire environments. The model is based on a porous media description that includes the effects of gas transport within the laminate along with swelling. Model comparisons are conducted against the data from Quintiere et al. Simulations are conducted for both coupon level and intermediate scale one-sided heating tests. Comparisons of the heat release rate (HRR) as well as the final products (mass fractions, volume percentages, porosity, etc.) are conducted. Overall, the agreement between the available data and model is excellent considering the simplified approximations to account for flame heat flux. A sensitivity study using a newly developed swelling model shows the importance of accounting for laminate expansion for the prediction of burnout. Excellent agreement is observed between the model and data of the final product composition that includes porosity, mass fractions and volume expansion ratio.
\end{abstract}

KEYWORDS: modeling, heat release rate, carbon epoxy laminate.

\section{NOMENCLATURE LISTING}

$\begin{array}{llll}a_{p} & \text { pre-exponential rate coefficient }(1 / \mathrm{s}) & V^{*} & \text { mapped space element volume } \\ C_{P} & \text { specific heat }(\mathrm{J} / \mathrm{kg} \cdot \mathrm{K}) & W & \text { quadrature weight } \\ d_{f} & \text { weave thickness }(\mathrm{m}) & X & \text { dimension into laminate }(\mathrm{m}) \\ E_{a} & \text { activation energy }(\mathrm{kJ} / \mathrm{mol}) & Y & \text { species mass fraction } \\ h & \text { sensible enthalpy }(\mathrm{kJ} / \mathrm{g}) & \text { Greek } & \\ h & \text { convection coefficient }\left(\mathrm{kW} / \mathrm{m}^{2} \cdot \mathrm{K}\right) & \alpha & \text { reaction progress variable } \\ H R R & \text { heat release rate }\left(\mathrm{kW} / \mathrm{m}^{2}\right) & \varphi & \text { volume fraction } \\ \Delta h_{c} & \text { heat of combustion }(\mathrm{kJ} / \mathrm{g}) & \rho & \text { density }\left(\mathrm{kg} / \mathrm{m}^{3}\right) \\ J & \text { Jacobian } & \zeta & \text { mapped space coordinate } \\ k & \text { thermal conductivity }\left(\mathrm{W} / \mathrm{m}^{2} \cdot{ }^{\circ} \mathrm{C}\right) & \tau & \text { mass ratio of char to resin } \\ K & \text { permeability }\left(\mathrm{m}^{2}\right) & \mu & \text { mass ratio of fiber }+ \text { char to initial matrix } \\ m, M & \text { mass }(\mathrm{kg}) & \Omega, \Omega^{\mathrm{e}} & \text { solution, element domains } \\ m,,, & \text { mass source } / \mathrm{sink}^{2} \text { term }\left(\mathrm{kg} / \mathrm{m}^{3} \cdot \mathrm{s}\right) & \text { subscripts } \\ m, & \text { mass flux }\left(\mathrm{g} / \mathrm{m}^{2} \cdot \mathrm{s}\right) & a d & \text { adiabatic } \\ p & \text { pressure }(\mathrm{Pa}) & o, e & \text { initial, end state } \\ q, & \text { heat flux }\left(\mathrm{kW} / \mathrm{m}^{2}\right) & f, c, g, r & \text { fiber, char, gas, resin } \\ R & \text { gas constant }(\mathrm{J} / \mathrm{mol} \mathrm{K}) & \infty & \text { far-field property } \\ T & \text { temperature }(\mathrm{K}) & \mathrm{L}, \mathrm{R} & \text { left, right } \\ t & \text { time }(\mathrm{s}) & i & \text { constituent, } i=\{c, f, g, r\} \\ V & \text { volume }\left(\mathrm{m}^{3}\right) & i & \text { element node }\end{array}$

\section{INTRODUCTION}

Composite polymer matrix materials such as carbon-epoxy are heavily being used in the aerospace industry due to their high strength to weight ratio and corrosion resistance. When exposed to fire, however, these materials degrade, releasing volatile gases, and producing char, resulting in a significant reduction in structural strength [1]. What is unique for this class of materials is the importance of modeling the internal dynamics of internally generated pyrolysis gases as they escape and burn, which in turn, increase the heat transfer to the surface. Thermal models that take into account the gas dynamics are therefore necessary to 
understanding the thermal response of these materials. From a fire safety standpoint the primary metrics of interest are the overall heat release rate and flame spread rate.

One of the earliest one-dimensional transient thermal models for polymer materials is that developed by Henderson et al. [2-4]. They modeled a glass-phenolic system as composed of either virgin or burnt (char) material. A global $n^{\text {th }}$ order Arrhenius model of resin decomposition was proposed and calibrated with their burn loss measurements. Bulk averaged transport properties were modeled using a reaction progress variable expressed in terms of the time-dependent matrix density. The thermal equations were solved using an implicit finite difference method. Good agreement was observed between predictions and experimental data of temperature and gas pressure.

Dimitrienko systematically developed more advanced thermo-mechanical models for high-temperature composites over a series of studies [5-10]. In his approach, the matrix is assumed to consist of fiber, resin, char and gas. The formulation of the system of thermal and mechanical equations are derived using asymptotic averaging [6] yielding a hierarchy of linearized equations. The first and second order systems of equations are used to develop local solutions for idealized periodic matrix configurations [10]. These solutions are then used to determine the functional relations for bulk constitutive relations [10] that are calibrated from experimental data [7]. The numerical approach used by Dimitrienko was largely based on finite-difference methods.

More recently, Luo and DesJardin developed a thermo-mechanical model for composite materials using a homogenization approach for both the thermal and mechanical fields resulting in a system of equations that are self-consistent [11]. Predictions of temperature and pressure fields of chopped glass and phenolic composites compared well with the previous experiments of Henderson [3].

For the current study, the model of Luo and DesJardin is extended to examine carbon-epoxy composite laminates. The thermal, transport, and kinetic properties for the carbon-epoxy matrix are developed from property data given by the study of Quintiere, Walters and Crowley (QWC) [12]. In their study, an extensive set of experiments was conducted to characterize the flammability of aircraft carbon-fiber laminates These measurements included data for thermal gravimetric analysis (TGA) and differential scanning calorimetry (DSC) to construct phenomenological pyrolysis decomposition kinetics and intermediate scale one-sided heating and flame spread tests.

The rest of this study is organized as follows. A brief overview of the thermal model is first provided with emphasis on a new volumetric swelling model that accounts for both changes in porosity as well as a description of the finite element (FE) algorithm used to expand the laminate thickness. Computations of the response of coupon level samples are first conducted and compared to the mass loss and volumetric expansion data of QWC. Simulations of one-sided heating tests are then performed to match those of QWC. Comparisons are conducted of both the heat release rate (HRR) as well as the final products (mass fractions, volume percentages, porosity, etc.). Finally conclusions are drawn as to findings from this study.

\section{THERMAL MODELING}

The material modeling for this effort is based on homogenization theories developed for both the thermal and mechanical fields of composite systems by DesJardin et al. [11]. In this approach, the local governing equations within each constituent (e.g., fiber, resin, gas, char, etc.) are first defined. These equations, representing the local mass, thermal and mechanical response of that material, are assumed to be locally valid within a given constituent. These equations are then averaged over a localized volume. The volume is chosen to be sufficiently large relative to the mesoscopic features of the laminate, (e.g., a unit cell associated with the weave), but small relative to the system, i.e., the entire laminate structure. After averaging the transport equations, additional surface integral terms appear in the equations representing the interphase processes. These terms originate from commuting the averaging operator with differentiation and, in general, are unknown and problem specific. Experimental data, specific to the material studied, is required to determine the interphase process terms.

The gas density and individual gas species are tracked through the phase averaged species mass conservation equations. The total number of gaseous species depends upon the complexity of the pyrolysis model. Phase-averaged species conservation equations are solved for the bulk density, $\rho_{G}$, and the mass fraction of the $i^{\text {th }}$ gas constituent, $Y_{i, s}$, 
$\partial\left(\phi_{g} \rho_{g}\right) / \partial t=\nabla \cdot\left(\rho_{g} \underset{\sim}{K} \cdot \nabla p_{g} / \mu_{g}\right)+\dot{m}_{v o l}^{\prime \prime \prime} \phi_{g}+\dot{m}_{i n t}^{\prime \prime \prime}$

$\partial\left(\phi_{g} \rho_{g} Y_{i, g}\right) / \partial t=\nabla \cdot\left(\rho_{g} Y_{i, g} \underset{\sim}{\boldsymbol{K}} \cdot \nabla p_{g} / \mu_{g}\right)+\nabla \cdot\left[\rho_{g} \phi_{g} D_{i m} \nabla Y_{i, g}\right]+\dot{m}_{i, v o l}^{\prime \prime \prime} \phi_{g}+\dot{m}_{i, i n t}^{\prime \prime \prime}$

where a Darcy's Law is employed to approximate the bulk gas transport, i.e., $\phi_{g} \vec{u}_{g}=-\boldsymbol{K} \cdot \nabla p_{g} / \mu_{g}$ requiring the specification of the permeability, $\boldsymbol{K}$. Fick's Law of diffusion accounts for the effects of differential diffusion, i.e., $\vec{u}_{i, d i f f}=-\left(D_{i m} \nabla Y_{i, g}\right) / \tilde{Y}_{i, g}$. The term, $\dot{m}_{v o l}^{\prime \prime \prime}=\sum \dot{m}_{i, v o l}^{\prime \prime \prime}$, on the RHS accounts for the production or consumption of species from volumetric reactions within the gas phase (e.g., oxidation of pyrolysis gases within the material). The last term, $\dot{m}_{i n t}^{\prime \prime \prime}=\sum \dot{m}_{i, \text { int }}^{\prime \prime}$, accounts for the production of pyrolysis gases from endothermic decomposition and evaporation processes which occur at phase interfaces. For the solid phases, the species conservation equation simplifies once the density of a given phase is assumed to be constant, i.e., $\rho_{i, s} \partial \phi_{i, s} / \partial t=\dot{m}_{i, \text { int }}^{\prime \prime \prime}$. Thermal equilibrium among the solid, liquid and gas phases is assumed resulting in a single transport equation to describe energy transport,

$$
\begin{aligned}
\rho C_{P} \partial T / \partial t & \left.=\nabla T \cdot\left[\rho_{g} C_{P, g} \underset{\sim}{\boldsymbol{K}} \cdot \nabla p_{g} / \mu_{g}\right]+\nabla \cdot \underset{\sim}{\boldsymbol{k}} \cdot \nabla T\right)+\sum_{i=1}^{N_{g}}\left(\nabla T \cdot C_{P, i} \rho_{g} \phi_{g} D_{i, m} \nabla Y_{i, g}\right) \\
& -\sum_{i=1}^{N} h_{i}\left(\phi_{g} \dot{m}_{i, \text { vol }}^{\prime \prime \prime}+\dot{m}_{i, \text { int }}^{\prime \prime \prime}\right)
\end{aligned}
$$

where $\left.\rho\left(=\sum \rho_{i} \phi_{i}\right)\right)$ and $C_{P}\left(=\sum \phi_{i} \rho_{i} / \rho C_{P, i}{ }^{\prime}\right.$ are the bulk density and specific heat, respectively. The terms on the RHS of Eq. 3 represent bulk advection (via. a Darcy Law approximation), conduction, differential diffusion, and oxidation/decomposition rate processes.

\section{Pyrolysis Rate Modeling}

For the purposes of constructing phenomenological Arrhenius based decomposition rates, a reaction progress variable, $\alpha$, is often introduced and is defined in terms of the solid mass, $m\left(=m_{r}+m_{f}+m_{c}\right)$, as,

$\alpha=\frac{m-m_{o}}{m_{e}-m_{o}}$.

The subscript $o$ and $e$ represent the initial and final states of the solid, respectively. Assuming the fiber does not participate in the pyrolysis then the rates of decomposition of the resin $(r)$, char $(c)$ and gas $(g)$ can be expressed directly in terms of changes in $\alpha$,

$\dot{m}_{r, \text { int }}^{\prime \prime \prime}=-\frac{1}{1-\tau}\left[\rho_{o}-\left(V_{e} / V_{o}\right) \rho_{e}\right] \dot{\alpha}$

$\dot{m}_{c, \text { int }}^{\prime \prime \prime}=\frac{\tau}{1-\tau}\left[\rho_{o}-\left(V_{e} / V_{o}\right) \rho_{e}\right] \dot{\alpha}$

$\dot{m}_{g, \text { int }}^{\prime \prime \prime}=\left[\rho_{o}-\left(V_{e} / V_{o}\right) \rho_{e}\right] \dot{\alpha}$

where $\dot{\alpha}$ is modeled using an Arrhenius rate law given by QWC as: $\dot{\alpha}=\frac{1-\alpha}{1-\mu} k\left(T^{\prime}\right.$, with $\mu=\left(m_{f}+m_{c}\right)_{e} / m_{o}$ being defined as the char fraction and $k$ given as $k=a_{p} \exp \left(-E_{a} / R T\right)$. Values for the activation energy $\left(E_{a}\right)$ and pre-exponential constant $\left(a_{p}\right)$ were determined by QWC using TGA data resulting in kinetic parameters of $E_{a}=182 \mathrm{~kJ} / \mathrm{mol}$ and $a_{p}=9.67 \times 10^{10} \mathrm{~s}^{-1}$. The quantity $V_{e} / V_{c}$ in Eq. 5 is the overall volumetric expansion ratio assuming complete charring and is set equal to a value of 2.2 to match the measurements of QWC. The quantity $\tau$ is the mass of char produced per unit mass of resin and is defined as:

$\tau \equiv \frac{\dot{m}_{c, i n t}^{\prime \prime \prime}}{\dot{m}_{r, i n t}^{\prime \prime \prime}}=\frac{V_{e}}{V_{o}} \frac{\left(\phi_{c} \rho_{c}\right)_{e}}{\left(\phi_{r} \rho_{r}\right)_{o}}$ 
which can be directly related to the residue fraction as,

$\tau=\frac{\rho_{o} \mu-\left[\rho_{f} \phi_{f}\right]_{o}}{\left[\phi_{r} \rho_{r}\right]_{o}}$

where $\rho_{o}, \rho_{f}$ and $\phi_{f, c}$ are given by QWC as $1530 \mathrm{~kg} / \mathrm{m}^{3}, 1230 \mathrm{~kg} / \mathrm{m}^{3}$ and 0.6 , respectively. Neglecting the mass of the initial gas then the initial fiber volume fraction is $\phi_{r, o}=0.398$ and gas volume fraction is $\phi_{g, o}=1-\phi_{r, o}-\phi_{f, o}=0.007$. Substituting the initial volume fractions and densities into Eq. 6 results in $\tau=0.17$ which is consistent with the reported value given by QWC of $0.20+0.05$. It is important to note that for modeling purposes, the values of $\mu$ and $\tau$ should be self-consistent.

\section{Matrix Thermal Properties}

One of the major modeling challenges is accurate models of the transport coefficients $\boldsymbol{K}$ and $\boldsymbol{k}$. In the current study, the conductivity of the composite is modeled using a curve fit to the data of QWC given as: $k=0.023 \mathrm{~T}^{0.46} \mathrm{~W} / \mathrm{m}^{2} \cdot{ }^{\circ} \mathrm{C}$, where $T$ is measured in ${ }^{\circ} \mathrm{C}$.

The gas flow through the matrix is modeled as that of flow through a bank of circular cylinders for which the permeability is be estimated using the following correlation [13].

$\underset{\sim}{\boldsymbol{K}}=K_{\perp} \underset{\sim}{\boldsymbol{I}}$

where $\boldsymbol{I}$ is the unity tensor and $K_{\perp}$ represents the permeability associated with flow across a periodic bank of tubes with diameter, $d_{f}$, given as,

$K_{\perp}=\frac{\phi_{g}^{3} d_{f}^{2}}{C\left(1-\phi_{g}\right)^{2}}$

where $C=144$ if the tubes are cylindrical in shape and $d_{f}$ is set equal to $0.1 \mathrm{~mm}$ as an estimate of the fiber toe diameter. The toe diameter is estimated by dividing the thickness of the laminate $(3.2 \mathrm{~mm})$ by twice the total number of plies (16). The rationale being that each layer is composed of overlapping toes in the weave and the resin rich region between layers is small relative to the weave thickness. The resulting initial and final permeability using this approach are $2.42 \times 10^{-17} \mathrm{~m}^{2}$ and $2.83 \times 10^{-10} \mathrm{~m}^{2}$, respectively, however, the upper bound of $K_{\perp}$ is clipped to a value of $1 \times 10^{-13}$ to avoid unnecessarily small time steps to maintain numerical stability. For the heat flux ranges used in this study, the pressure time history is insensitive to the value chosen for this upper clipping value as long as it is larger than $1 \times 10^{-14}$.

The bulk specific heat of the matrix is modeled using a reaction progress variable description, $C_{P}(T, \alpha)=C_{P, o}(T)+\alpha\left(C_{P, e}(T)-C_{P, o}(T)\right.$, , using temperature dependent virgin $C_{P, o}(T)$, and charred properties $\left(C_{P, e}(T)\right)$ from $\mathrm{QWC}$ given as,

$C_{P, o}=0.75+0.0041 T \mathrm{~J} / \mathrm{kg} \cdot \mathrm{K}$

$C_{P, e}=0.84+0.0035 T \mathrm{~J} / \mathrm{kg} \cdot \mathrm{K}$

where $T$ is in ${ }^{\circ} \mathrm{C}$.

\section{Composite Swelling}

As discussed by QWC, an overall volumetric expansion ratio of $V_{e} / V_{o}=2.2$ is observed in the experiments. As will be shown in the results, the effects of volumetric expansion has a pronounced effect on burn-out times because of the changes in gas volume fraction and the overall growth of the composite thickness. To 
account for these effects, an evolution equation for the $i^{\text {th }}$ solid volume fraction can, in principle, be derived,

$\frac{D \phi_{i}}{D t}=\frac{\dot{m}_{i, i n t}^{\prime \prime \prime}}{\rho_{i}}+\dot{V}_{i, e x p}$

where the subscript $i(=f, r, c)$, represents either fiber, resin or char and the corresponding source/sink term, $\dot{m}_{i, i n t}^{\prime \prime \prime}$, is given in Eq. 5. The second term on the RHS of Eq. 12 accounts for the increasing solid volume fraction from swelling (expansion) processes and can be directly related to the divergence of the $i^{\text {th }}$ solid material velocity, i.e., $\dot{V}_{i, e x p}=-\phi_{i} \nabla \cdot \vec{u}_{i}$. To rigorously account for the effects of swelling requires a detailed analysis of the mechanical response of the structure to determine $\vec{u}_{2}$ that, in turn, will depend on the thermal field (via, thermal expansion, fiber fraying, etc.). To model this coupled thermo-mechanical system, a presumed micro-mechanics description is often defined in the context of unit cell homogenization approaches [11]. The exact nature of the swelling process is, however, quite complicated and potentially difficult to validate experimentally therefore a simpler phenomenological approach is pursued where the solid volume fraction is directly expressed as a linear function of the reaction progress variable,

$\phi_{i}=\phi_{i, o}+\left(\phi_{i, f}-\phi_{i, o}\right) \alpha$

where the initial $\left(\phi_{i, o}\right)$ and final $\left(\phi_{i, f}\right)$ volume fractions of each solid constituent that are summarized in Table 1 below.

Table 1. Summary of initial and final volume fractions of solid constituents.

\begin{tabular}{|c|c|c|}
\hline $\boldsymbol{i}$ & $\phi_{i, o}$ & $\phi_{i, f}$ \\
\hline $\mathrm{f}$ & 0.600 & 0.273 \\
\hline $\mathrm{r}$ & 0.393 & 0.000 \\
\hline $\mathrm{g}$ & 0.007 & 0.706 \\
\hline $\mathrm{c}$ & 0.000 & 0.021 \\
\hline
\end{tabular}

This simple model accounts for the leading order effects from composite swelling, which is to decrease the solid volume fractions and increase the gas volume fraction that will be shown to be important in the results. In addition, the composite geometry change from swelling is also accounted for using a newly developed element expansion algorithm. In this approach, each element node located at position $\vec{x}_{o}$ is first assigned a neighboring reference node, $\vec{x}_{o, r e f}$. The distance between these nodes at the start of the simulation is defined as: $\vec{d}_{o}=\vec{x}_{o}-\vec{x}_{o, \text { ref }}$ as shown in Fig. 1 . As the expansion process proceeds the distance between the two nodes is directly related to the expected volumetric expansion, $V / V_{c}$, via, $\vec{d}=\vec{d}_{o} V / V_{o}$, where $V / V_{c}$ can be determined by re-arranging Eq. 4 ,

$\frac{V}{V_{o}}=\frac{\left(\rho_{e} V_{e} / V_{o}-\rho_{o}\right) \alpha+\rho_{o}}{\rho}$

resulting in a non-linear dependence of $V / V_{c}$ on $\alpha$. In Eq. 14, $\rho=\sum \phi_{i} \rho_{i}$ is the total matrix density that is computed at each node. The neighboring reference node associated with each moving node is selected by a closest search in a direction that is opposite to that of a user prescribed expansion direction, $\hat{n}_{e x p}=-\vec{d}_{o} / \mid \vec{d}_{o}$. The user specified direction could, in general, be selected to point anywhere but for this study is chosen to point towards the boundary that is heated (i.e., the left boundary in Fig. 1). Element expansion is implemented after each integration time step by moving the element nodes as: $\vec{x}(t)=\vec{x}_{r e f}(t)+\vec{d}(t)$. The reference node shown in Fig. 1, is in turn, a function of its own reference node, and so on for the rest of the nodes. At the boundary farthest from the heating source, the reference node and node of interest are the same, therefore $\vec{d}_{o}=\overrightarrow{0}$ and so the nodes farthest from the heated boundary do not move (i.e., the right boundary in Fig. 1). Ideally, the nodes farthest away from the heating source should be updated first and the nodes closest to the heating source updated last. However, because of the unstructured nature of the data in the FE implementation, it is not straightforward to implement such an update strategy. 
Alternatively, a time linearization procedure is implemented such that, $\vec{x}(t+\Delta t)=\vec{x}_{r e f}(t)+\vec{d}(t+\Delta t)$, where $\vec{d}(t+\Delta t)$ is determined as $\vec{d}(t+\Delta t)=\vec{d}_{o} V(t) / V_{o}$ with $V / V_{c}$ evaluated using Eq. 14. The resulting algorithm yields a smooth movement of nodes so that $V / V_{o} \rightarrow V_{e} / V_{c}$ as $\alpha \rightarrow 1$.

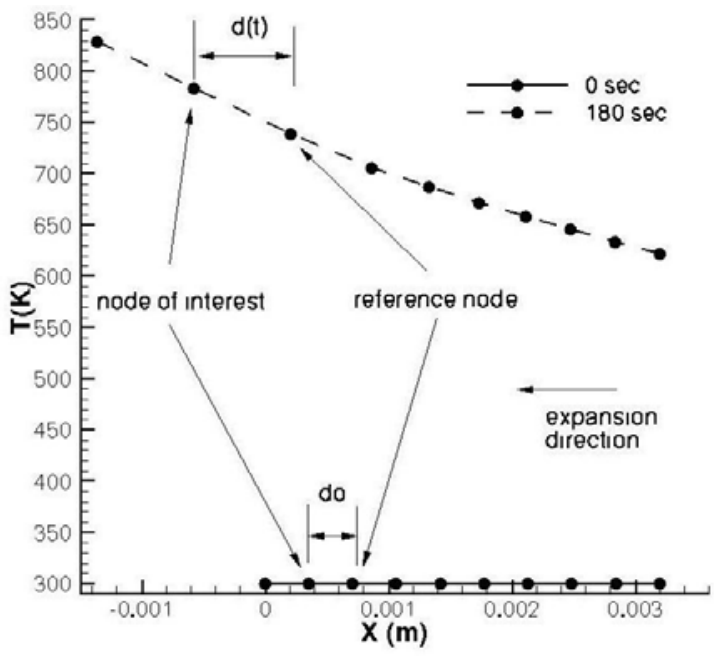

(a)

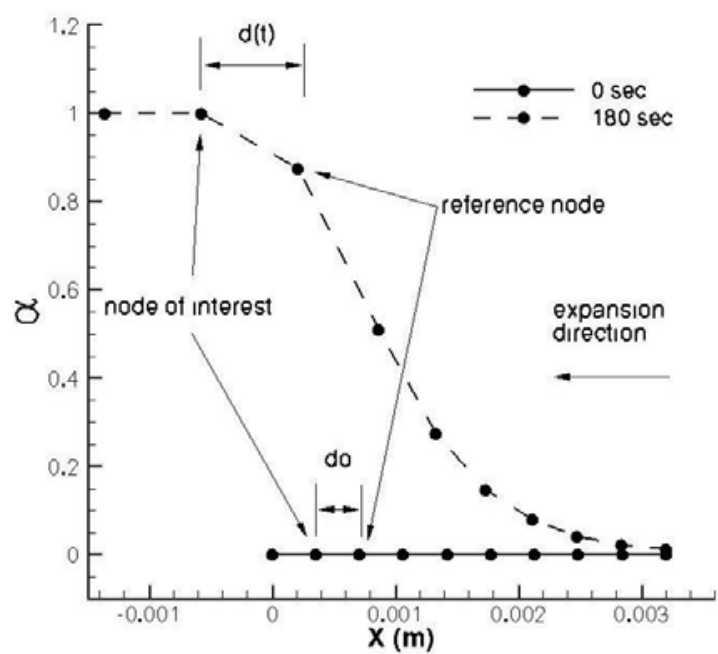

(b)

Fig. 1. Element expansion process in 1-D showing (a) temperature and (b) reaction progress variable at $t=0 \mathrm{~s}$ and $180 \mathrm{~s}$.

Figure 1 illustrates the element expansion algorithm for one-sided heating of a $3.2 \mathrm{~mm}$ thick carbon-epoxy laminate. The computations are one-dimensional using 10 elements across the thickness. The right boundary is assumed adiabatic. A constant $60 \mathrm{~kW} / \mathrm{m}^{2}$ heat flux is imposed on the left boundary. The initial temperature (Fig. 1a) and $\alpha$ (Fig. 1b) are shown and after $3 \mathrm{~min}$ of heating. As expected, the temperature on the left face of the laminates increases for which decomposition of the laminate occurs as shown by the increase in $\alpha$. The expansion direction $\hat{n}_{e x x}$ denoted in the figures is directed to the left as that is direction of the applied heat. For illustration purposes, the second node in from the left boundary is identified as the node of interest and its self-identified reference node is the neighboring node to the right. As shown, the distance between them $(\vec{d})$ grows with time in accordance with Eq. 14 such that $|\vec{d}| / \mid \vec{d}_{o}$ approaches a final value of 2.2 corresponding to $V / V_{c}$.

\section{Gas Properties and Exothermic Chemical Reactions}

The mixture of pyrolysis gases and air are treated as ideal gases using CHEMKIN polynomial fits for thermodynamic properties [14]. The proportions of carbon, hydrogen and oxygen in the pryolysis gas are assumed to be the same as those given by Tewarson as $\mathrm{CH}_{1.3} \mathrm{O}_{0.2}$ [15]. For lack of data, the sensible enthalpy and specific heats of the pyrolysis product gas are assumed to be that of methane $\left(\mathrm{CH}_{4}\right)$ since the molecular weights are similar (i.e., 16.5 vs. 16). Once the pyrolysis gas leaves the composite, it is assumed to burn according to the following one-step molar reaction.

$$
\mathrm{CH}_{1.3} \mathrm{O}_{0.2}+1.225\left(\mathrm{O}_{2}+3.76 \mathrm{~N}_{2}\right) \longrightarrow \mathrm{CO}_{2}+0.65 \mathrm{H}_{2} \mathrm{O}+1.225(3.76) \mathrm{N}_{2}
$$

Using a suggested heat of combustion of $\Delta h_{C}=28.8 \mathrm{~kJ} / \mathrm{g}$ by Tewarson [15], a calculated heat of formation for the pyrolysis gas using Eq. 15 is $h_{C H_{1.3} O_{0.2}}^{o}=-4.5785 \mathrm{~kJ} / \mathrm{g}$ resulting in an adiabatic flame temperature of $T_{a d}=2300 \mathrm{~K}$.

\section{Numerical Solution Approach}

Equations 1-3 are solved using a finite element (FE) method. The application of FE methods to the conservation equations requires expressing said equations in a weak form and discretizing over elements. 
The weak forms of equations are solved using a standard Galerkin FE formulation utilizing high-order Lagrange interpolating polynomials using an $\alpha$-family of time integration schemes [16]. Further details of the FE implementation can be found in Ref. [11].

\section{RESULTS}

To explore the limitations of the thermal model, two sets of data are used from QWC. The first are coupon scale samples for which TGA data is available at several heating rates. The second set of model validation runs considered are one-sided heating calorimeter tests carried out by QWC. In these tests, $15 \times 15 \mathrm{~cm}$ samples are heated by a radiant heater. The experiments are carried out according to Title 14 Code of Federal Regulations (CFR) 25.853 a-1, which is the Federal Aviation Administration (FAA) flammability test method for large surface area materials for aircraft cabin interiors. Reported values of heat release rate (HRR), final volume expansion ratio $\left(V / V_{c}\right)$, thickness expansion ratio, final porosity $\left(V_{g} / V_{\epsilon}\right)$, char + resin mass to initial resin mass ratio $\left(Y_{r c}=\left(M_{c}+M_{r}\right)_{e} / M_{r, c}\right)$ and fraction of remaining mass $\left(Y_{r c f}\right)$ are given by QWC in Figs. 12, 22 and 27 of their report, respectively.

\section{Coupon Scale Validation}

The heating rates selected for the simulations are $1,3,10$ and $30{ }^{\circ} \mathrm{C} / \mathrm{min}$ to match those of QWC and compare the sample mass fraction to the TGA data. The bulk solid mass fraction, $Y_{r c f}=M_{s} / M_{s, o}=\left(M_{r}+M_{c}+M_{f}\right) / M_{s, o}$, is computed by integrating the masses of each constituent element-wise $\left(\Omega^{e}\right)$ over the solution domain $(\Omega)$ using the following relation,

$M_{i}=\int_{\Omega} \phi_{i} \rho_{i} d V \simeq \sum_{e} \int_{\Omega^{e}} J(\vec{\zeta}) \phi_{i}(\vec{\zeta}) \rho_{i}(\vec{\zeta}) d V^{*}=\sum_{e} \sum_{j} W_{j}\left(\vec{\zeta}_{j}\right) J\left(\vec{\zeta}_{j}\right) \phi_{i}\left(\vec{\zeta}_{j}\right) \rho_{i}\left(\vec{\zeta}_{j}^{\prime}\right.$

where $J$ is the time varying Jacobian associated with the mapping of the physical space $(\vec{x})$ to the reference computational space $(\vec{\zeta})$ as the elements stretch due to swelling expansion processes. The quantities $W_{j}$ and $\vec{\zeta}_{\text {J }}$ are the quadrature weights and locations in mapped space on the element for the numerical integration. One-dimensional simulations are conducted using prescribed time dependent temperature boundaries. The temperatures on the boundaries are increased in time to be consistent with the prescribed heating rate. A total of 10 elements are used to discretize the $3.2 \mathrm{~mm}$ thickness sample which is deemed adequate based on a grid sensitivity check (not shown).

Figure 2a shows instantaneous snapshots of temperature (solid lines) and $\alpha$ (dashed lines) for the $10{ }^{\circ} \mathrm{C} / \mathrm{min}$ heating case at $t=775,1775$ and $2025 \mathrm{~s}$. The temperature is nearly uniform indicating that a thermally lumped analysis for developing the kinetic rates is valid. Figure $2 \mathrm{~b}$ shows the solid mass fraction compared to TGA results for heating rates of $3{ }^{\circ} \mathrm{C} / \mathrm{min}, 10^{\circ} \mathrm{C} / \mathrm{min}$ and $30^{\circ} \mathrm{C} / \mathrm{min}$. As shown, the overall agreement is excellent - demonstrating that the Arrhenius kinetics, thermal properties and heat of decomposition source/sink terms are properly incorporated into the framework. 


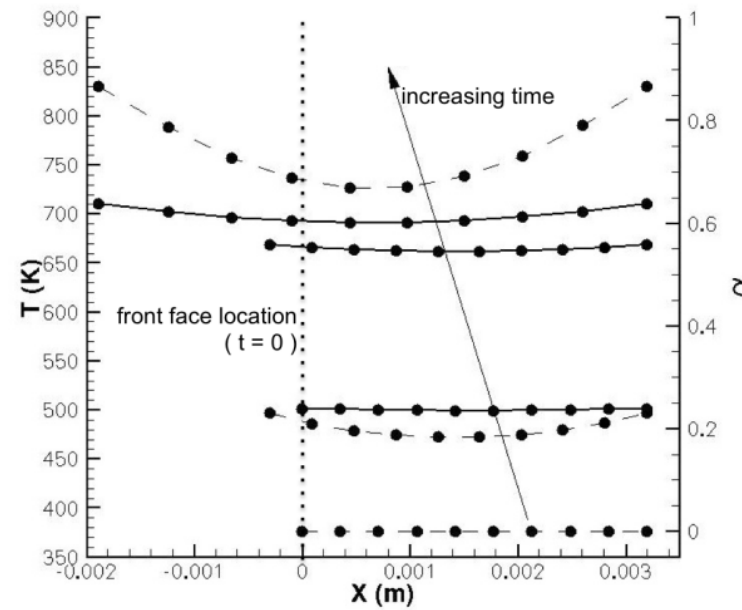

(a)

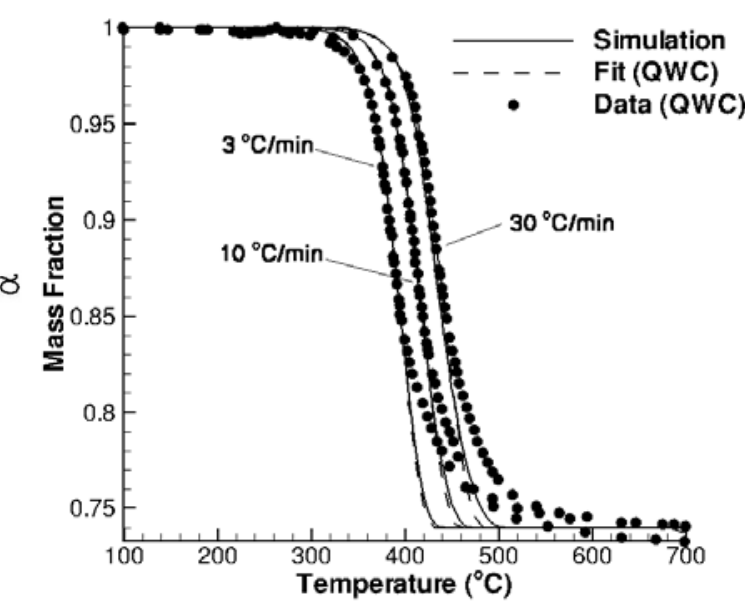

(b)

Fig. 2. Coupon scale simulations showing: (a) instantaneous snapshots of temperature (solid lines) and $\alpha$ (dashed lines) at $t=775,1775$ and $2025 \mathrm{~s}$ for a $10{ }^{\circ} \mathrm{C} / \mathrm{min}$ heating rate; (b) comparison of predictions of solid mass fraction (solid lines) to TGA data from Quintiere et al. (symbols) and their curve fit using a firstorder Arrhenius rate model.

\section{Intermediate Scale Validation using One-sided Heating Experiments}

To compare against the data, one-dimensional simulations are conducted using 30 elements for grid independent results. Measured bulk quantities of the final products are directly computed in the FE model by integrating over the entire solution domain similar to that for the coupon level samples. To estimate the heat release rate, it is assumed that the mass flux blowing off of the heated surface instantly burns with the surrounding air. The heat release rate per unit surface area may then be estimated as: $H R R=\left.\dot{m}_{g}^{\prime \prime}\right|_{L} \Delta h_{C}$, where $\left.\dot{m}_{g}^{\prime \prime}\right|_{L}$ is the mass flux from the heated left surface of the FE model.

The thermal boundary conditions for the left $(\mathrm{L})$ and right $(\mathrm{R})$ boundaries are treated by defining the net heat flux in terms of incident $(i)$, convective and surface blowing.

$\dot{q}_{L}^{\prime \prime}=\dot{q}_{i}^{\prime \prime}+h\left(T_{\infty, L}-T_{L}\right)-\left.\dot{m}_{g}^{\prime \prime} h_{g}\right|_{L}$

$\dot{q}_{R}^{\prime \prime}=h\left(T_{\infty, R}-T_{R}\right)-\left.\dot{m}_{g}^{\prime \prime} h_{g}\right|_{R}$

where $\dot{q}^{\prime \prime}=-\vec{q} \cdot \hat{n}$ and $\dot{m}_{g}^{\prime \prime}=\overrightarrow{\dot{m}}_{g}^{\prime \prime} \cdot \hat{n}$ with $\hat{n}$ being the outward normal unit vector from the solid surface. The convective coefficients for the left and right boundaries are assumed to be constant and equal to $h=10$ $\mathrm{kW} / \mathrm{m}^{2} \cdot \mathrm{K}$. The far-field temperature on the right surface, $T_{\infty, R}$, is assumed constant and equal to $300 \mathrm{~K}$.

The far-field temperature on the left surface is also assumed to be equal to $300 \mathrm{~K}$ until $\left.\dot{m}_{g}^{\prime \prime}\right|_{L}>\dot{m}_{c r i t}^{\prime \prime}=1.0$ $\mathrm{g} / \mathrm{m}^{2} \cdot \mathrm{s}$. At this point, ignition is assumed to occur and the far-field temperature is raised to $T_{\infty, L}=0.5\left(T_{a d}+T_{L}\right)$ to account for the additional heat flux from the near-wall flame. $T_{a d}=2300 \mathrm{~K}$ is a calculated adiabatic flame temperature using the one-step reaction given in Eq. 15 . The value of $\dot{m}_{c r i t}^{\prime \prime}$ is selected to be $1 / 10$ of the estimated mass flux based on the heat release rates given by QWC and serves as a trigger to increase the heat flux from near-wall burning. After sufficient burning, the $\left.\dot{m}_{g}^{\prime \prime}\right|_{L}$ again falls below $\dot{m}_{c r i t}^{\prime \prime}$ and the flame is assumed to extinguish. When this occurs the left far-field temperature is reset to $T_{\infty, L}=300 \mathrm{~K}$. For the gas transport, the gas density is determined on the left boundary assuming a constant

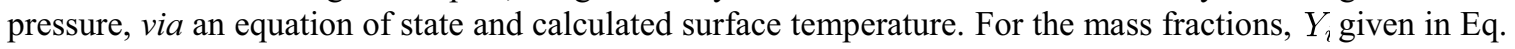
2 , the following outflow convective boundary condition is imposed. 
where $u_{g, n}=\vec{u}_{g} \cdot \hat{n}$. The specification of the boundary conditions for gas transport on the right boundary is less certain. While aluminum foil was used to seal the back surfaces in the experiments, it is not clear that it provided an effective seal since tests with and without the foil showed little difference in the resulting flame observed jetting out the front face [12]. To explore the sensitivity of the calculations to this uncertainty, cases are conducted using both open (i.e., constant pressure and the convective boundary of Eq. 19 and closed (sealed) right boundaries. For the closed cases the flux for the gases on the right boundary are set equal to zero.

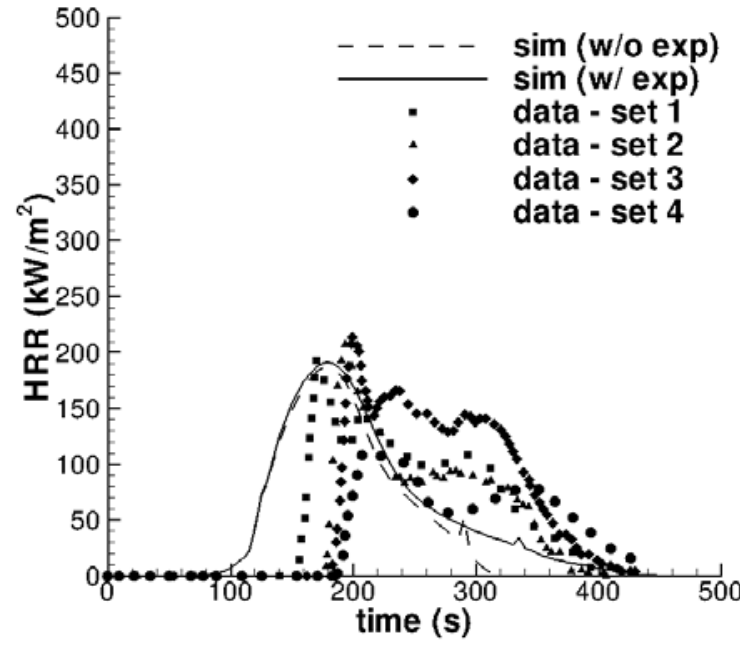

(a)

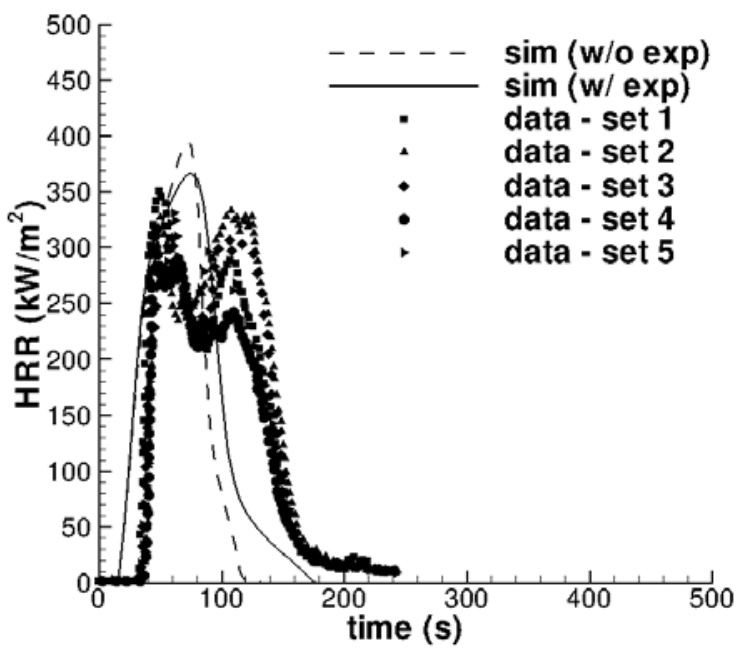

(c)

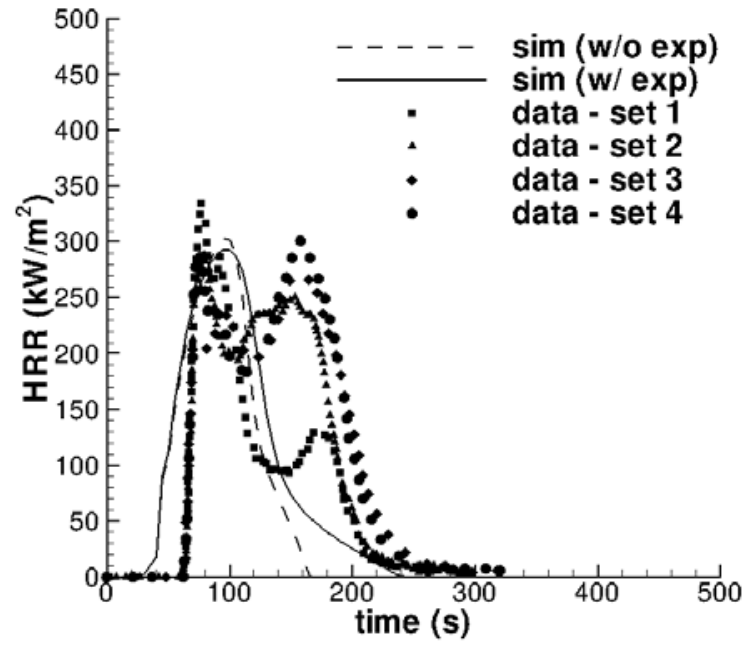

(b)

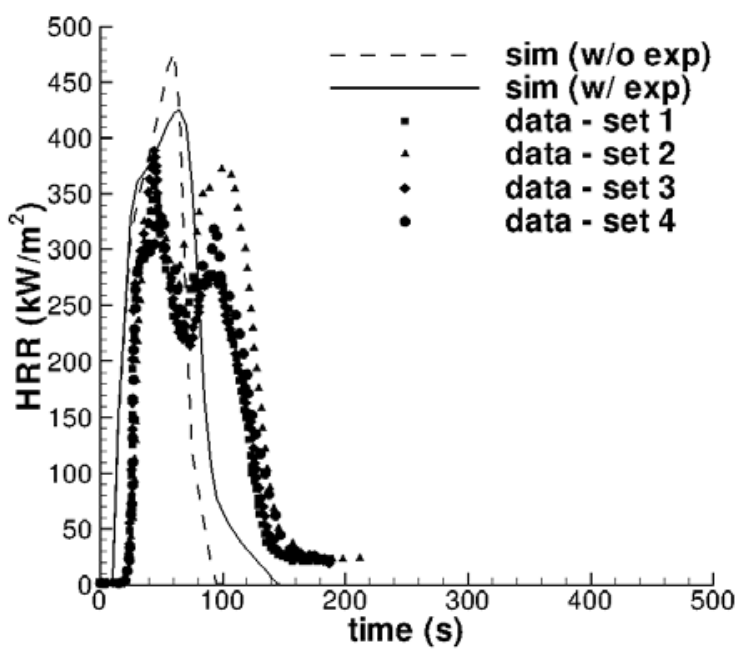

(d)

Fig. 3. One-sided heating cases with open right boundary showing heat release rate (HRR) comparisons for incident heat fluxes of: (a) $25 \mathrm{~kW} / \mathrm{m}^{2}$; (b) $50 \mathrm{~kW} / \mathrm{m}^{2}$; (c) $75 \mathrm{~kW} / \mathrm{m}^{2}$; (d) $100 \mathrm{~kW} / \mathrm{m}^{2}$.

Figure 3 shows comparisons of the heat release rate for four different heating rates of (a) $\dot{q}_{i}^{\prime \prime}=25$, (b) 50 , (c) 75 and (d) $100 \mathrm{~kW} / \mathrm{m}^{2}$. For all cases, the right boundary is assumed open. For each heat flux case, simulations are conducted both with (solid lines) and without (dashed lines) the expansion model activated and are compared to data (symbols) consisting of four to five separate runs. Overall the agreement of the model to the data is reasonable considering the simplifications in estimating the heat release rate and the variability in the experimental data. In all cases, the model under-predicts ignition times with errors ranging from $50 \%$, for the low heat flux cases, to $30 \%$ for the higher heat flux cases. This is to be expected using 
the indicator of ignition based on the very simplified model of HRR for which all of the mass leaving the composite is assumed to instantly burn. In reality, a critical mass of pyrolysis gas is first required to form a premixed ignition kernel. The subsequent burning of pyrolysis gases will then be controlled by near-wall turbulent mixing processes. These processes, however, are not accounted for in the current model. Comparing simulation cases without (w/o) and with (w/) the expansion model shows that accounting for volumetric expansion processes extends the burnout time by at least $50 \%$ for all cases resulting in much better agreement to the data. The reason for the premature burnout times w/o expansion is due to the effective reduced mass of the sample by using the final volume fractions summarized in Table 1 without increasing the overall size of the sample. As expected, a smaller mass then significantly shortens the burnout time.

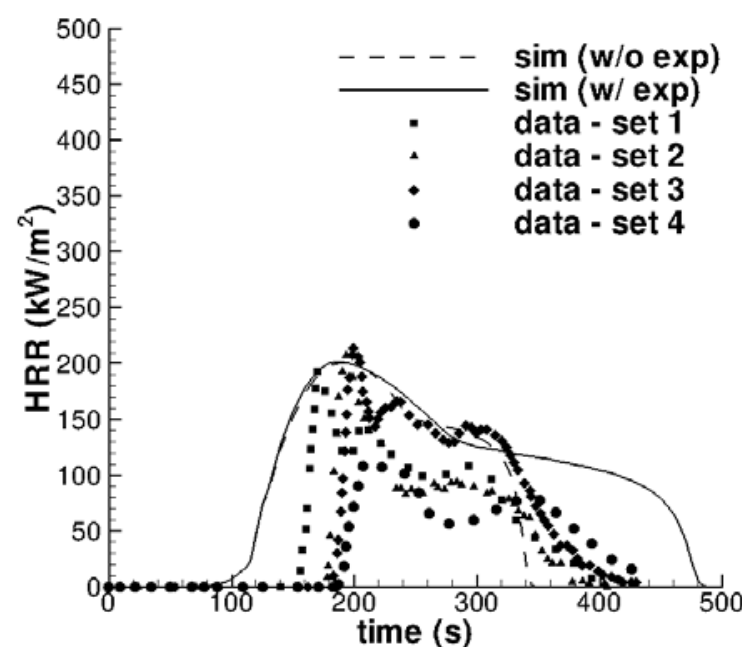

(a)

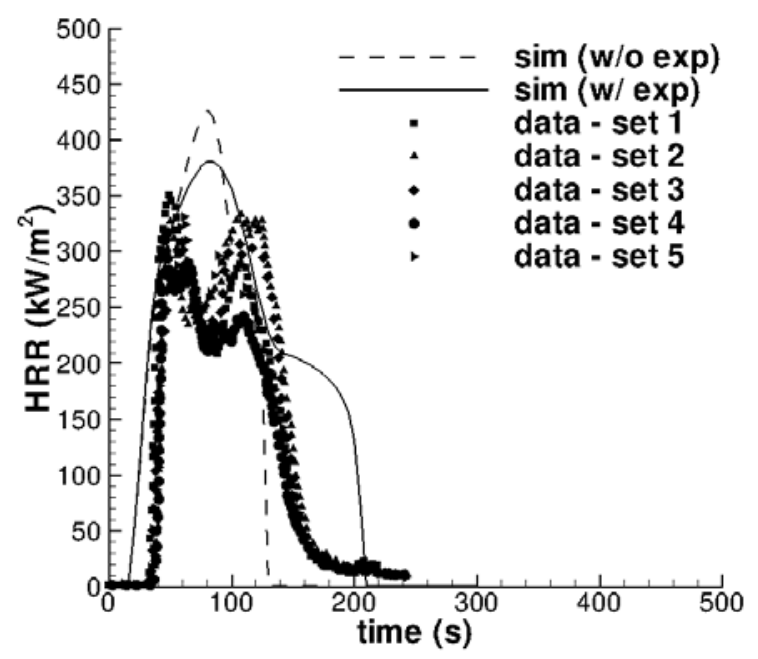

(c)

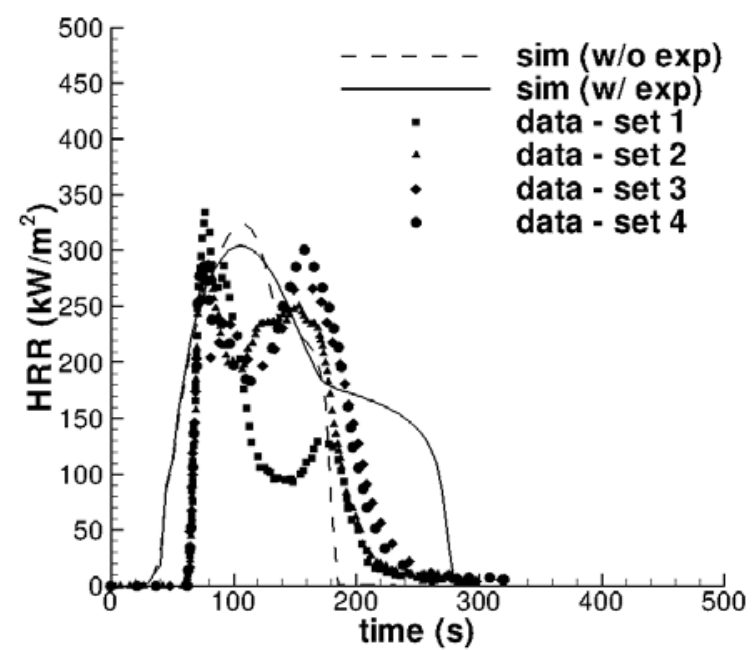

(b)

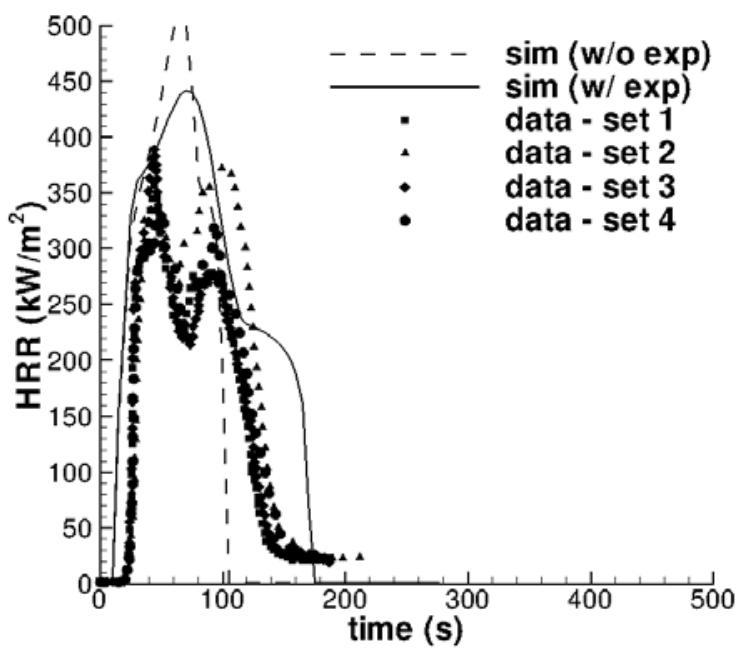

(d)

Fig. 4. One-sided heating cases with sealed right boundary showing heat release rate (HRR) comparisons for incident heat fluxes of: (a) $25 \mathrm{~kW} / \mathrm{m}^{2}$; (b) $50 \mathrm{~kW} / \mathrm{m}^{2}$; (c) $75 \mathrm{~kW} / \mathrm{m}^{2}$; (d) $100 \mathrm{~kW} / \mathrm{m}^{2}$.

While the peak heat flux and burn out times predicted are reasonable in Fig. 3, the duration and overall heat release, $\Delta Q=\int_{t} H R R(t) d t$, are significantly smaller than the experiments. This may be expected since the gas escaping out the right boundary was not accounted for in the overall heat release. To explore this possibility, additional simulations are conducted sealing the right boundary. Figure 4 are the same cases as those of Fig. 3 but with the right boundary sealed. It is apparent that the sealing of the right boundary has a pronounced effect on $\Delta Q$ as well as the time history of HRR. A secondary plateau in heat release is observed that is more consistent with the double peak HRR history observed in the experiments. Since the 
simulations with the expansion model with open (Fig. 3) and sealed (Fig. 4) appear to bound the HRR data, it is reasonable to assume that the actual boundary from the experiments lies between these limits. This observation shows the importance of properly characterizing the unheated boundary for model validation purposes - the easiest approach may be to simply leave the unheated surface completely open or insulated with a very porous thermal blanket (e.g., superwool).

QWC offer an explanation for the twin peak HRR in Figs. 3 and 4. They attribute the appearance of the first peak to the composite seeking to achieve a steady-state thermal distribution for the resin binder as an insulation layer is built up and the second peak to a thermal heating wave reaching the back of the composite and being inhibited thereafter from the insulation backer board. A more quantitative complementary explanation can be explored by examining the instantaneous distributions of through thickness temperature and pressure. Figure 5 shows snapshots of (a) $T \& \alpha$ and (b) $p_{g} \& K_{\perp}$ at $t=25,50$ and $150 \mathrm{~s}$.

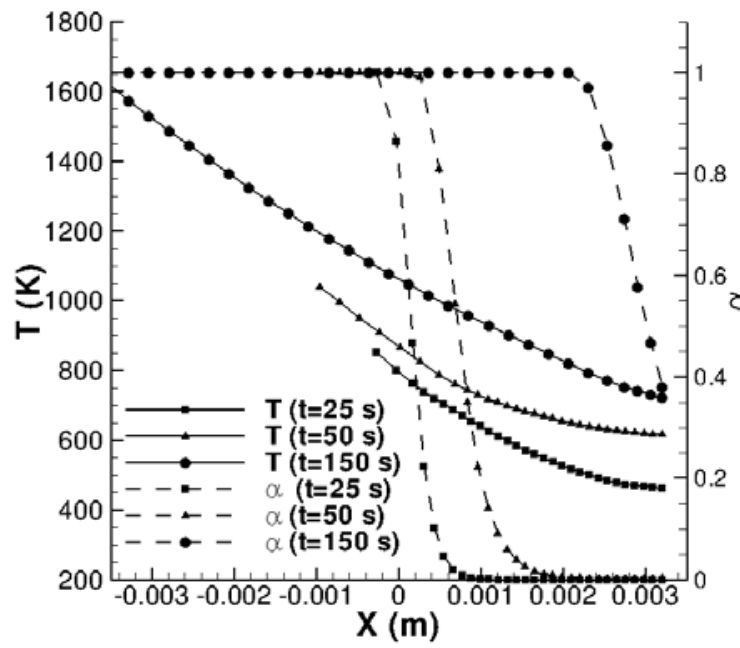

(a)

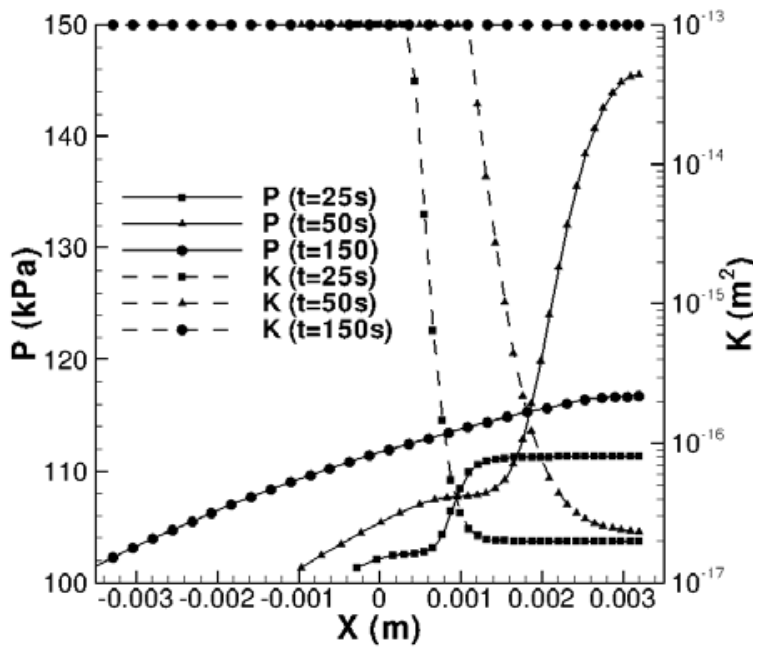

(b)

Fig. 5. Instantaneous snapshots of: (a) $T \& \alpha$; (b) $p_{g} \& K_{\perp}$ at $t=25,50$ and 150 s. Symbols denote position of FE nodes.

The temperature smoothly rises with increasing time as the decomposition front penetrates deeper into the laminate (denoted by $\alpha$ ). The gas pressure, however, shows a very different behavior with a local peak pressure at the decomposition front where the permeability is low. The pressure continues to grow with time to a peak value of $1.5 \mathrm{~atm}$ until the decomposition front reaches the back face of the laminate at $t=75 \mathrm{~s}$. Between $75 \mathrm{~s}$ and $150 \mathrm{~s}$ the residual resin decomposes resulting in a capacitance of pressure that slowly vents off creating the plateau region of Fig. $4 \mathrm{~d}$.

While the current model qualitatively captures the double peak history, the predicted second peak in HRR is lower than measured in the experiments. The reasons for this are two-fold. The first is the simplified estimate of HRR that does not account for the effects of turbulent mixing already discussed. Future fully coupled simulations will explore relaxing this assumption. The second reason is the limitations of the current permeability model.

The exact time history of the venting process out the front face will be very sensitive to how the permeability is modeled. As shown in Fig. 5b, the model for permeability results in a fairly monotonic permeability profile through the decomposition front (even though it is a strong non-linear function of porosity, see Eq. 9. If, however, the permeability decreases abruptly in the charred region due fiber clumping and/or collapse then the gas could potentially be limited enough to cause the more pronounced dip in HRR observed in the data. To account for these effects would require a much more sophisticated permeability model that depends on mechanical response. Nonetheless, the final product volume and mass fractions are well predicted as shown in Fig. 6 showing comparisons of (a) $V / V_{c}, V_{g} / V_{\epsilon} \& Y_{r c}$ and (b) $Y_{r c f}$ to data from QWC that are summarized in Figs. 22 and 27 of their report using the simulation results with the sealed right boundary. The overall agreement for $V / V_{c}, V_{g} / V_{\epsilon} \& Y_{r c}$ is quite good with errors less than 
$15 \%$ over the entire range of incident heat fluxes considered. The final mass predictions show in Fig. $6 \mathrm{~b}$ appear to under-predict the data at lower heat fluxes, however considering the repeatability uncertainty in the data, it is difficult to draw definitive conclusions about the accuracy of the modeling

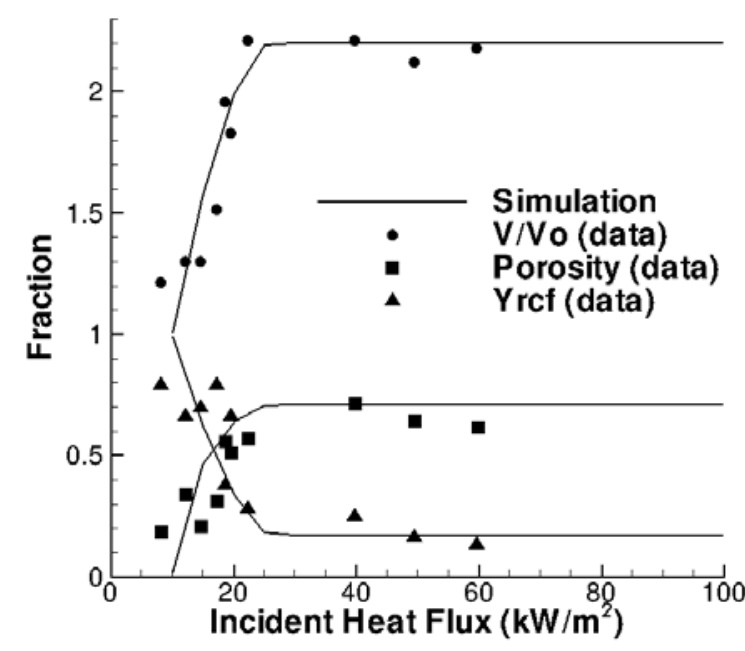

(a)

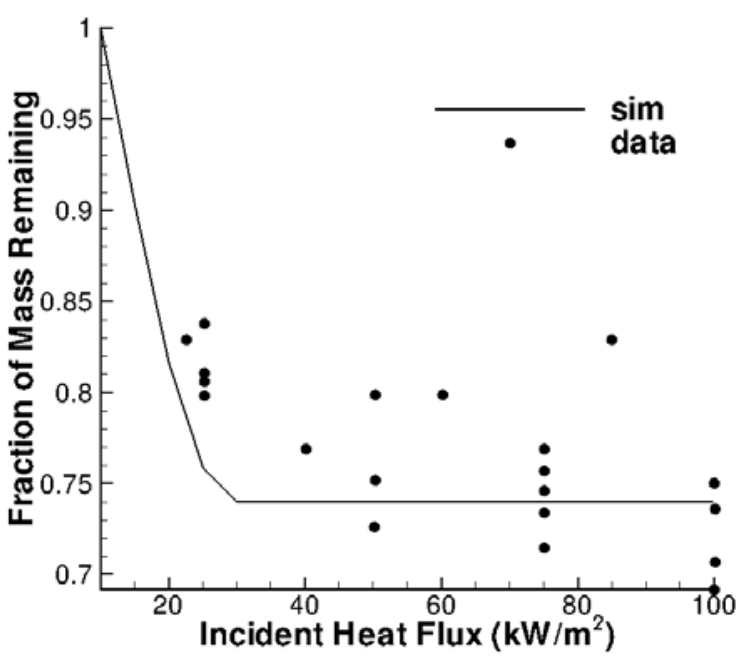

(b)

Fig. 6. Comparisons of predictions of: (a) $V / V_{c}, V_{g} / V_{\epsilon} \& Y_{r c}$; (b) $Y_{r c f}$ to data with increasing incident heat flux.

\section{CONCLUSIONS}

A thermal model for a carbon-epoxy laminate is developed based on the data of Quintiere et al. [12]. The model includes pyrolysis decomposition, heat and mass transport, and volumetric swelling using a novel finite element expansion algorithm. Model validation runs are conducted using TGA and one-sided heating experiments. Overall good agreement is observed between the model and data. Neglecting the effects of the composite swelling resulted in significant under-predictions of flame burnout - highlighting the importance of accounting for laminate swelling for the current epoxy-carbon material. Remaining discrepancies in HRR predictions are attributed to three factors. The first is the uncertainty as to the specification of the unheated back boundary for the gas transport. Simulation results bound the HRR data using either an open or perfectly closed boundary indicting that the experimental conditions lie someplace in-between. This emphasizes the importance of being able to accurately characterize this boundary for future validation level experiments. The second factor is the simplified estimate of HRR using the decoupled calculations that do not account for important gas-phase turbulent combustion processes. The third factor is the permeability model that appears to be monotonic through the decomposition front and therefore does not account for potentially importance matrix collapse processes that may change the time history of the HRR. However, the overall heat release predicted by the model seems to be in qualitative agreement with the data. Lastly, comparisons of predictions to measurement of final volumetric expansion ratio, porosity and final char mass is quite good indicating that the overall thermal modeling approach is sound.

\section{ACKNOWLEDGEMENTS}

This research is supported by that National Science Foundation (NSF) under Grant CBET-1033328 and Sandia National Laboratories. Sandia National Laboratories is a multi-program laboratory operated by Sandia Corporation, a wholly owned subsidiary of Lockheed Martin Company, for the US Department of Energy's National Nuclear Security Administration under contract DE-AC04-94AL85000.

\section{REFERENCES}

[1] Mouritz, A.P., Feih, S., Kandare, E., Mathys, Z., Gibson, A.G., DesJardin, P.E., Case, S.W., Lattimer B.Y., (2009) Review of Fire Structural Modelling of Polymer Composites, Composites. 40: 1800-1814, http://dx.doi.org/10.1016/j.compositesa.2009.09.001 
[2] Florio, J., Henderson, J.B., Test, F.L., Hariharan, R., (1991) A study of the effects of the assumption of local-thermal equilibrium on the overall thermally-induced response of a decomposing, glass-filled polymer composite, International Journal of Heat and Mass Transfer 34(1): 135-147, http://dx.doi.org/10.1016/0017-9310(91)90181-D

[3] Henderson, J.B., Wiebelt, J.A., Tant, M.R., (1985) A model for the thermal response of polymer composite materials with experimental verification, Journal of Composite Material 19: 579-595, http://dx.doi.org/10.1177/002199838501900608

[4] Henderson, J.B., Wiecek, T.E., (1987) A mathematical model to predict the thermal response of decomposing expanding polymer composites, Journal of Composite Materials, 21: 373-393, http://dx.doi.org/10.1177/002199838702100406

[5] Dimitrienko, Y.I., (2000) Thermomechanical behavior of composites under local intense heating by irradiation, Composites: Part A, 31: 591-598, http://dx.doi.org/10.1016/S1359-835X(99)00094$\underline{9}$

[6] Dimitrienko, Y.I., (1998) Mechanics of porous media with phase transformations and periodical structures 1. Method of asymptotic averaging, European Journal of Mechanics -A/Solids,. 17(2): 305-319, http://dx.doi.org/10.1016/S0997-7538(98)80088-0

[7] Dimitrienko, Y.I., (1997) Thermomechanical behavior of composite materials and structures under high temperatures: 1. Materials, Composites Part A, 28: 453-461, http://dx.doi.org/10.1016/S1359-835X(96)00144-3

[8] Dimitrienko, Y.I., (1997) Thermomechanical behavior of composite materials and structures under high temperatures: 2. Structures, Composites Part A, 28: 463-471, http://dx.doi.org/10.1016/S1359-835X(96)00145-5

[9] Dimitrienko, Y.I., (1997) Internal heat-mass transfer and stresses in thin-walled structures of ablating materials, Int. J. Heat Mass Transfer, 40(7): 1701-1711, http://dx.doi.org/10.1016/S00179310(96)00149-4

[10] Dimitrienko, Y.I., (1998) Mechanics of porous media with phase transformations and periodical structures 2. Solutions of local and global problems, European Journal of Mechanics -A/Solids, 17(2): 321-337, http://dx.doi.org/10.1016/S0997-7538(98)80089-2

[11] Luo, C., DesJardin, P.E., (2007) Thermo-mechanical damage modeling for a glass-fiber phenolicresin composite material, Composites Science and Technology, 67: 1475-1488, http://dx.doi.org/10.1016/j.compscitech.2006.07.030

[12] Quintiere, J.G., Walters, R.N., Crowley, S., "Flammability Properties of Aircraft Carbon-Fiber Structural Composite," U.S. Department of Transportation, Federal Aviation Administration: Washington, DC. 2007, pp. 1-34.

[13] Kuwahara, F., Kameyama, Y., Yamashita, S., Nakayama, A., (1998) Numerical Modeling of Turbulent Flow in Porous Media using a Spatially Periodic Array, J. of Porous Media,. 1: 47-55.

[14] Kee, R.J., Rupley, F.M., Miller, J.A., "The CHEMKIN Thermodynamic Data Base," Sandia National Laboratory: Livermore, CA, 1987

[15] Tewarson, A., "Generation of Heat and Chemical Compounds in Fires", The SFPE Handbook of Fire Protection Engineering, DiNenno, P.J. (ed.,) National Fire Protection Association. 2002, p. $3 / 82-3 / 161$.

[16] Reddy, J.N., Finite Element Method. 1993, New York, NY: McGraw Hill. 